\title{
Sequential Development of Electrical and Chemical Synaptic Connections Generates a Specific Behavioral Circuit in the Leech
}

\author{
Antonia Marin-Burgin, ${ }^{\star}$ F. James Eisenhart, ${ }^{\star}$ Serapio M. Baca, William B. Kristan Jr, and Kathleen A. French \\ Section of Neurobiology, Division of Biological Sciences, University of California, San Diego, La Jolla, California 92093-0357
}

\begin{abstract}
Neuronal circuits form during embryonic life, even before synapses are completely mature. Developmental changes can be quantitative (e.g., connections become stronger and more reliable) or qualitative (e.g., synapses form, are lost, or switch from electrical to chemical or from excitatory to inhibitory). To explore how these synaptic events contribute to behavioral circuits, we have studied the formation of a circuit that produces local bending (LB) behavior in leech embryos. This circuit is composed of three layers of neurons: mechanosensory neurons, interneurons, and motor neurons. The only inhibition in this circuit is in the motor neuron layer; it allows the animal to contract on one side while relaxing the opposite side. LB develops in two stages: initially touching the body wall causes circumferential indentation (CI), an embryonic behavior in which contraction takes place around the whole perimeter of the segment touched; one or $2 \mathrm{~d}$ later, the same touch elicits adult-like LB. Application of bicuculline methiodide in embryos capable of LB switched the behavior back into CI, indicating that the development of GABAergic connections turns CI into LB. Using voltage-sensitive dyes and electrophysiological recordings, we found that electrical synapses were present early and produced CI. Inhibition appeared later, shaping the circuit that was already connected by electrical synapses and producing the adult behavior, LB.
\end{abstract}

Key words: invertebrate; development; gap junction; synapse; behavior; imaging

\section{Introduction}

Effective operation of any neuronal circuit depends on having correct synaptic connections. Although much attention has been focused on the development of chemical synapses, there is a growing appreciation that electrical synapses also play important roles in neuronal circuits (Galarreta and Hestrin, 1999; Gibson et al., 1999; Bennett, 2000a,b; Deans et al., 2001; Long et al., 2004) and in their development (Roerig and Feller, 2000; Drapeau et al., 2002; Tresch and Kiehn, 2002). In many systems, neurons form transient electrical synapses around the time of chemical synapse formation (Peinado et al., 1993a; Penn et al., 1994), suggesting either that transient electrical synapses may be necessary for the formation of chemical synapses (Fischbach, 1972; Szabo et al., 2004) or that they may contribute to activity-dependent refinement of chemical synapses by coordinating activity between coupled neurons (Kandler and Katz, 1995). These proposed developmental mechanisms can best be tested by following a single neuron from its first synaptic contacts through to its adult set of

Received Nov. 23, 2004; revised Jan. 21, 2005; accepted Jan. 23, 2005.

This work was supported by National Institutes of Health Research Grants MH43396 and NS35336 to W.B.K., a Fundacion Antorchas grant to A.M.-B., and National Institute of General Medical Sciences Training Grant T32AG00216 to F.J.E. We thank Panvera for supplying the dyes. We thank Pablo E. Schilman for assistance with statistical analyses and comments on this manuscript.

${ }^{*}$ A.M. B. and F.J.E. contributed equally to this work.

Correspondence should be addressed to Dr. Antonia Marin-Burgin, 3119 Pacific Hall, University of California, San Diego (0357), La Jolla, CA 92093-0357. E-mail: aburgin@ucsd.edu.

F. J. Eisenhart's present address: Microsoft Corporation, 810 West Emerson Street, Seattle, WA 98119.

D0I:10.1523/JNEUROSCI.4787-04.2005

Copyright $\odot 2005$ Society for Neuroscience $\quad 0270-6474 / 05 / 252478-\bullet \$ 15.00 / 0$ connections. Recent work on fish (Drapeau et al., 2002) and rodents (Bonnot et al., 2002) suggests that such an approach may in the future be possible in a vertebrate CNS but invertebrate nervous systems, with their identified neurons (Muller et al., 1981; Marder and Calabrese, 1996), provide the means for cell-by-cell analysis now.

We have used voltage-sensitive dyes and intracellular recordings to investigate the development of identified neurons and their synaptic connections in the leech CNS. We focused on the development of a motor circuit that produces a segmental behavior, local bending (LB). Localized touch to the body wall elicits LB, a longitudinal contraction on the side touched and simultaneous relaxation of the opposite side (Kristan, 1982). Embryonic behaviors in Hirudo medicinalis appear in sequence, beginning with spontaneous behaviors (lateral ridge formation and bends of the germinal plate), at $51 \%$ of embryonic development (ED), and followed by mechanically evoked behaviors [circumferential indentation (CI), avoidance, and local bend] starting at 55\% ED (see Fig. 1 A). The LB behavior appears at 60\% ED and replaces an embryonic behavior called CI that appears at 55\% ED (Reynolds et al., 1998b) and is characterized by a contraction of the longitudinal muscles on both sides of the midline of the germinal plate after a light touch, no matter which side was stimulated (see Fig. 1A).

LB is produced by a three-layered neuronal circuit: four mechanosensory neurons (P-cells) excite $\sim 17$ LB interneurons (LBI), which excite $\sim 16$ pairs of inhibitory and excitatory longitudinal motor neurons (see Fig. $1 B, C$ ) (Lockery and Kristan, 1990; Lewis and Kristan, 1998). The only known lateral connec- 
Table 1. Responses to moderate pressure delivered to a mid-body segment in leeches at different developmental stages

\begin{tabular}{llllr}
\hline & $\begin{array}{l}\text { Number of intact leeches } \\
\text { that produced } \mathrm{Cl} \text { in } \\
\text { control solution }\end{array}$ & $\begin{array}{l}\text { Number of intact leeches } \\
\text { that produced Cl in BMI } \\
\text { solution }\end{array}$ & $\begin{array}{l}\text { Number of dissected body } \\
\text { wall preparations that } \\
\text { produced Cl in BMI }\end{array}$ & Total \\
\hline $52-55 \%$ ED & 10 of 10 & 10 of 10 & 3 of 3 & 13 of 13 \\
$60-70 \%$ ED & 0 of $8^{a}$ & 5 of 8 & 2 of 3 & 7 of 11 \\
$>100 \%$ ED & 0 of $5^{a}$ & 5 of $5^{b}$ & 5 of $5^{b}$ & 10 of 10 \\
\hline
\end{tabular}

${ }^{a}$ Animals older than $60 \% \mathrm{ED}$ produced $\mathrm{LB}$ in response to touch when tested in saline solution.

${ }^{b}$ These leeches were nearly intact, but a small incision was made at the anterior end to facilitate drug access to the ventral nerve cord.

coated Petri dishes. Drug-treated animals were soaked in BMI saline for $2 \mathrm{~h}$ before testing. During testing, we stimulated each leech with the mechanical probe every $10 \mathrm{~min}$ and scored the elicited behavior. We recorded several representative samples of the responses using a C-Mounted Hitachi KP-M1 monochrome CCD camera (Image Labs International, Bozeman, MT). Because we were looking for qualitative changes in the behavior, and because the time it took for the drug to take effect varied among animals, we report only the percentage of times that each behavior was elicited imme-

tions in the circuit are inhibitory and are made among the motor neurons. These motor neurons make both electrical and chemical synapses with one another. Synergistic excitatory motor neurons are electrically coupled (Ort et al., 1974), and inhibitory motor neurons (Stuart, 1970; Ort et al., 1974; Sawada et al., 1976) also centrally inhibit the excitatory motor neurons to those same muscles (Ort et al., 1974; Granzow and Kristan, 1986) through GABAergic synapses (Cline, 1983, 1986) (see Fig. 1C).

The data presented here strongly suggest that the LB circuit is initially established by electrical connections that produce CI transiently with the mature form of LB appearing only after inhibitory chemical synapses form.

\section{Materials and Methods Animals}

Adult medicinal leeches $(H$. medicinalis) were purchased from a commercial supplier [Leeches USA (Westbury, NY) or Carolina Biological Supply (Burlington, NC)] and maintained in a breeding colony in our laboratory. Embryos were released from their cocoons no earlier than $9 \mathrm{~d}$ after egg deposition and were then held at $20-24^{\circ} \mathrm{C}$ in "embryo water," sterile-filtered Arrowhead spring water (Arrowhead Water, Brea, CA) with $32 \mu \mathrm{mol}$ of $\mathrm{MgCl}_{2}$ and $40 \mu \mathrm{mol}$ of $\mathrm{CaCl}_{2}$ added per liter.

\section{Staging}

We determined the embryonic stage of each embryo using external morphological features that run from $0 \%$ (egg deposition) to $100 \%$ of ED (Reynolds et al., 1998a). Development proceeds at $\sim 3 \%$ ED per day at $20^{\circ} \mathrm{C}$. All experiments were performed in ganglia from the mid-body of the animal (segments 7-15) to minimize the effects of the anteriorposterior gradient of development in the leech; ganglia in this region differ by no more than 1\% ED (Reynolds et al., 1998a).

\section{Behavioral experiments}

Mechanical probe. A 1 inch length of 0.001 inch tungsten wire (California Fine Wire, Grover Beach, CA) was bent into a tight loop and attached to a wooden handle. We touched the body wall with the tip of this loop, at either the left or the right lateral edge. Animals were tested in control saline solution and then in a solution containing GABA antagonist. The same probe and the same force $(3-6 \mathrm{mN})$ were used in both cases. $\mathrm{LB}$ is produced by stimuli that excite the pressure-sensitive mechanoreceptor neurons in a single segment (Lockery and Kristan, 1990; Lewis and Kristan, 1998).

Drugs. Intact animals were studied in embryo water. Dissected animals were studied in normal leech saline consisting of the following (in $\mathrm{mm}$ ): $115 \mathrm{NaCl}, 4 \mathrm{KCl}, 1.8 \mathrm{CaCl}_{2}, 1.5 \mathrm{MgCl}_{2}, 10$ dextrose, 4.6 Tris maleate, and 5.4 Tris base, pH 7.4 (Muller et al., 1981). We used $0.5 \mathrm{~mm}$ bicuculline methiodide (BMI) (Sigma-Aldrich, St. Louis, MO) to block $\mathrm{GABA}_{\mathrm{A}}$ receptors. To allow better drug access, intact leeches were bathed in BMIcontaining embryo water after a small hole was made at the anterior end of the leech to open the circulatory system to the surrounding solution. During all dissections, the leeches were anesthetized in normal leech saline that contained $8 \%(\mathrm{v} / \mathrm{v})$ ethanol and then returned to embryo water or normal leech saline. The dissected animal was exposed sequentially to normal saline, to $0.5 \mathrm{~mm}$ BMI in normal saline, and back to normal saline.

Behavioral monitoring. Leeches were housed individually in Sylgard- diately after the $2 \mathrm{~h}$ drug treatment (Table 1 ).

Motion tracking in 100\% ED embryos. We recorded the image of the body wall preparation through the same CCD camera. Images were collected and digitized using a Scion LG-3 frame grabber card and image acquisition software (Scion, Frederick, MD) on a personal computer (PC). Previous studies had introduced an optic flow algorithm to characterize leech body wall movements from video recordings (Zoccolan and Torre, 2002); we used a different algorithm (Lucas and Kanade, 1981 ) that produces very similar motion estimates (Ye and Haralick, 2000).

Bend profile calculation. For each trial, we captured images at $30 \mathrm{~Hz}$ for $2.0-2.5 \mathrm{~s}$. These $60-75$ frames spanned the time before, during, and after stimulation. We calculated optic flow fields between successive frames. After calculating the movements of the entire body wall, we selected a rectangular region of interest (ROI) that showed robust movement and was free from edge or pinning artifacts. The ROI spanned one to two annuli along the long axis of the leech and included its entire circular axis. (An annulus is a raised ring of the leech body wall; there are five annuli per body wall segment.) For a given leech, we observed behavior in the same location for all trials, and we monitored movements produced primarily by the longitudinal muscles. Hence, for each pixel in the ROI, we extracted the component of the movement that ran parallel to the leech's long axis. The average movement at each circumferential location in the ROI created a profile of longitudinal movement between any two images. We smoothed these motion profiles with a Gaussian filter. For each trial, we calculated these motion profiles at each time point, generating a three-dimensional characterization of the local bend over time. Because the movement typically peaked at $\sim 1.5 \mathrm{~s}$ after stimulus onset and held steady for some time, we used the motion profile at $1.5 \mathrm{~s}$ as the bend profile that represented the bending response and called this the "bend profile." The units of displacement in bend profiles were originally pixels, which were then normalized to the number of pixels per annulus for each leech so that all displacements were ultimately expressed in terms of the number of annulus-widths traversed.

\section{Monitoring electrical activity with voltage-sensitive fluorescence resonance energy transfer dyes}

We removed a chain of five to eight ganglia from the embryo (always between ganglia 7 and 14), dissecting away the connective tissue capsule and glial coverings of the neurons, and stained the ganglia with fluorescence resonance energy transfer (FRET) dyes using a small modification of a previously described protocol (Taylor et al., 2003). We removed the connective tissue capsule from the dorsal surface of the ganglion and initially incubated it in a $10 \mu \mathrm{M}$ solution of the FRET donor $N$-(6-chloro7-hydroxycoumarin-3-carbonyl)-imyristoylphosphatidylethanolamine (CC2-DMPE) (Panvera, Madison, WI) (which is a coumarin-labeled phospholipid that comes dissolved in 100\% DMSO) plus $1.5 \mu \mathrm{l}$ of 20 $\mathrm{mg} / \mathrm{ml}$ pluronic F-127 in DMSO (Molecular Probes, Eugene, OR) in $3 \mathrm{ml}$ of saline. For the incubation, we pinned the ganglion in a Sylgard-coated dish and surrounded it with a $3 \mathrm{~mm}$ plastic cylinder sealed with petroleum jelly to make a watertight chamber. We then replaced the saline in the chamber with the staining solution for $30 \mathrm{~min}$ at room temperature, constantly recirculating the staining solution using a peristaltic pump (model RP-1; Rainin, Oakland, CA). After removing the first staining solution, we washed the tissue several times with fresh saline. We then incubated the ganglion with the FRET acceptor bis(1,3-diethyl- 
thiobarbiturate)-trimethine oxonol $\left[\operatorname{DiSBAC}_{2}(3)\right]$ (Panvera), which is an oxonol dye (Gonzalez and Tsien, 1995). We used a $20 \mu \mathrm{M}$ oxonol solution made by adding $3.6 \mu \mathrm{l}$ of $17 \mathrm{~mm} \mathrm{DiSBAC}_{2}(3)$ dissolved in $100 \%$ DMSO to $3 \mathrm{ml}$ of saline and bath-sonicating it for $3 \mathrm{~min}$. This solution remained in the chamber for the duration of the imaging. Leaving the oxonol in the bath did not increase background fluorescence because its extinction coefficient is small at $405 \mathrm{~nm}$ (the wavelength of the excitation light), and oxonol has 20-fold less fluorescent yield in aqueous solution than in cell membranes (Rink et al., 1980).

Optical recording. We acquired fluorescence images using an upright microscope (Axioskop 2FS; Zeiss, Thornwood, NY) equipped with a $40 \times, 0.8$ numerical aperture water-immersion objective (Acroplan; Zeiss). For epi-illumination, we used a tungsten halogen lamp (64625 HLX; Osram Sylvania, Danvers, MA) in standard housing (HAL 100; Zeiss), powered by a low-ripple power supply (JQE 15-12M; Kepco, Flushing, NY). For all voltage-sensitive dye imaging, we acquired images only at the coumarin emission wavelength. The filter set consisted of a $405 \pm 15 \mathrm{~nm}$ bandpass excitation filter, a $430 \mathrm{~nm}$ dichroic mirror, and a $460 \pm 25 \mathrm{~nm}$ bandpass emission filter (Chroma Technology Corporation, Brattleboro, VT). We acquired the optical data using a water-cooled CCD camera (MicroMax 512 BFT; Roper Scientific, Tucson, AZ) operated in frame-transfer mode at a frame rate of $20 \mathrm{~Hz}$. The CCD chip in this camera has $512 \times 512$ pixels, but we normally binned at $4 \times 4$ pixels, yielding a $128 \times 128$ pixel image. The quantum efficiency of the camera at the coumarin emission peak was $80 \%$. The CCD chip was maintained at $-25^{\circ} \mathrm{C}$ during imaging. Images were stored using the software package Win-View/32 (Roper Scientific, Trenton, NJ). We synchronized the optical and electrical recordings by feeding the frame timing signals emitted by the camera into the analog-to-digital board along with all the electrophysiological signals.

The combination of CC2-DMPE and oxonol yielded sensitivities of $5-20 \%$ change in fluorescence/100 $\mathrm{mV}$ for $1 \mathrm{~Hz}$ square-wave voltage signals with a $10 \mathrm{mV}$ amplitude, centered around a resting potential of $-50 \mathrm{mV}$. Much of the variation in sensitivity seemed to be attributable to differences in soma size, with larger somata giving higher sensitivity.

Analysis of images. We analyzed the images using Matlab (Mathworks, Natick, MA). We outlined the images of individual somata manually using a custom-made graphic user interface designed by Dr. Adam Taylor (Brandeis University, Waltham, MA). We averaged all pixels within each cellular outline in each frame, yielding a raw fluorescence signal for each cell. We eliminated the slow downward drift in the raw fluorescence images attributable to bleaching of the dyes by fitting the drift over the whole image with a mathematical function and subtracting a scaled version of it from all cell signals (Taylor et al., 2003). To find neurons connected to a particular neuron, we impaled the soma of the neuron with a microelectrode and passed current at $1 \mathrm{~Hz}$ while simultaneously collecting images at $20 \mathrm{~Hz}$ for $10 \mathrm{~s}$. We performed a coherence analysis to identify neurons with optical signals that were correlated with the stimulated one (Cacciatore et al., 1999).

\section{Electrophysiology}

Adults. Methods for adult experiments were similar to those described previously (Lockery and Kristan, 1990). Briefly, we dissected a single ganglion from the leech in normal leech saline (Muller et al., 1981), then dissected away the connective tissue and glial capsules covering the neuronal cell bodies. The ganglion was perfused continuously with fresh leech saline. We recorded on the stage of a compound microscope equipped with both transmitted light and fluorescence optics using sharp microelectrodes with resistances of 40-60 M $\Omega$ when filled with $3 \mathrm{M}$ potassium acetate, although we generally used a more dilute electrode solution [150 mm potassium gluconate (KG)] to minimize osmotic damage to cells. These latter electrodes had resistances of $\sim 200 \mathrm{M} \Omega$. Intracellular signals were recorded with an Axoclamp-2A amplifier (Axon Instruments, Foster City, CA) operating in current-clamp mode. We stimulated and acquired data using a PC-based system using custom software written in LabView 4.1 (National Instruments, Austin, TX).

Embryos. We obtained electrophysiological recordings from embryonic neurons in a similar manner; only the differences will be noted. The bathing solution for embryonic ganglia was a 1:1 mixture of L-15 culture medium (Invitrogen, Gaithersburg, MD) and leech saline. This medium was then diluted with distilled water, with the final concentration depending on the age of the embryo: a final concentration of $65 \%$ of the L- $15 /$ saline mixture for embryos at younger than $60 \% \mathrm{ED}, 70 \%$ for $60-$ $70 \%$ ED embryos, and $75 \%$ for $70-100 \%$ ED embryos. The $\mathrm{pH}$ of the solution was adjusted to 7.8 , because embryonic neurons at all stages had more robust electrophysiological properties at this slightly basic $\mathrm{pH}$. Control experiments using adult ganglia showed that both chemical and electrical synapses functioned normally in all the embryo media.

We removed a chain of five to eight ganglia from the embryo (between ganglia 7 and 14) and transferred it to a recording chamber, which was a $2 \times 3$ inch Sylgard-coated glass microscope slide onto which a $2-\mathrm{cm}-$ diameter rubber O-ring was glued. The chain of ganglia was pinned out, dorsal side up, by inserting 0.001-inch-diameter tungsten pins through the end ganglia and stretching slightly. Additional pins were bent like staples and used to crush the nerve cord between ganglia. This manipulation reduced movement artifacts caused by contractions of the muscles that run in the longitudinal connectives.

We recorded from neuronal somata using sharp microelectrodes that had resistances of 50-80 M $\Omega$ when filled with $3 \mathrm{M}$ KAc. Embryonic neurons proved to be extremely sensitive to osmotic changes, so we filled our electrodes with a solution of $120 \mathrm{~mm} \mathrm{KG}$, which is nearly iso-osmotic with the external medium; their resistance was 200-600 M $\Omega$. In addition, we added $0.1 \%$ Lucifer yellow to the electrode solution to help determine when a cell had been impaled and for morphological identification. To prevent photo damage, cells were illuminated only very briefly during recordings $(<1 \mathrm{~s})$. After recordings were complete, cells were viewed for longer periods and identified by morphological criteria (see below). When the low concentration of Lucifer yellow did not fill the neuron well enough to permit unambiguous identification, the neuron was reimpaled and filled with rhodamine-dextran (Molecular Probes), as described below.

Input resistance. To determine the input resistance of neurons, the same-sized current pulses $(-0.1 \mathrm{nA})$ were used to balance the bridge circuit and to measure the input resistance. Despite this precaution, we measured unreasonably high input resistances for both adult and embryonic neurons $(\sim 200 \mathrm{M} \Omega)$ when using our embryonic electrodes and intracellular solution. These values were much higher than those measured for adult neurons using the same electrodes filled with $3 \mathrm{~m} \mathrm{KAc}$ $(\sim 25 \mathrm{M} \Omega)$. This high apparent input resistance was probably caused by cellular debris that clogged the high-resistance electrode as it penetrated the cell. To compensate for this artifact, we normalized our embryonic input resistance data, multiplying it by the ratio of the mean input resistance measured for adult neurons measured with $3 \mathrm{~m}$ KAc divided by the value obtained using our embryonic intracellular solution; this ratio was 0.13 . We report only these normalized input resistance values.

Chemical IPSP amplitude. We measured the IPSP amplitude for chemical synapses by passing a current pulse into the presynaptic neuron while recording from the postsynaptic neuron (see Fig. 5). Because these connections are mediated in part by nonspiking release and generate sustained IPSPs with a slow onset time (Ort et al., 1974; Granzow et al., 1985), we used long-duration (1-3 s) pulses. Each pulse was preceded by a brief $(0.2 \mathrm{~s})$ hyperpolarizing "prepulse" $(-2 \mathrm{nA}$ for adults and $-0.5 \mathrm{nA}$ for embryos) to be sure that the membrane potential was initially below threshold for transmitter release. Depolarizing pulses of 1-6 nA were used for adult neurons and 0.5-1.5 nA for embryos, although many different pulse parameters generated the same IPSP size, presumably because the IPSPs reached the chloride reversal potential (Granzow and Kristan, 1986). We measured the IPSP size by subtracting the peak of the IPSP from the prestimulus baseline. IPSP sizes are reported as absolute values, although they represent hyperpolarizations.

Electrical coupling strength. In most cases, we measured the strength of an electrical synapse by passing a current ramp into one cell and recording the voltage response in the coupled cell. On a few occasions, we used a square current pulse (see Fig. 5), which gave identical results. To compute the coupling strength, we divided the voltage response in the coupled cell by the stimulus current. For ramp stimuli, we fit the postsynaptic $I-V$ curve with a straight line, using a custom program written in LabView; the slope of this line is the coupling strength. This quantity is 
often called the "transfer" or "mutual resistance" (Bennett, 1966), but we prefer to use the more heuristic term "coupling strength" and report our results in units of millivolt postsynaptic per nanoampere presynaptic rather than in megaohms.

The $I-V$ curves for nonrectifying electrical synapses were highly linear, whereas the slope for rectifying electrical synapses differed in the positive and negative portions of the stimulus ramp. We measured the coupling strength in both the forward direction (from cell A to cell B) and reverse direction ( $\mathrm{B}$ to $\mathrm{A}$ ). We characterized the overall coupling strength by the maximum of these values. In all cases, using the mean coupling strength, rather than the maximum value, yielded the same qualitative results.

Statistics. Statistical analyses were performed with SigmaStat 1.0 (SPSS, Chicago, IL) or Microsoft Excel 97 (Microsoft, Redmond, WA) on a PC running Windows NT 4.0. Comparisons between means were done with $t$ tests, although in all cases, results were confirmed with the nonparametric Mann-Whitney rank-sum test. Means are reported \pm SE.

\section{Results}

Behavioral switch from CI to LB

Early in development, localized touch elicits CI, a ring of longitudinal contraction that encircles the entire stimulated segment (Fig. 1A). Later in development, the same mechanical stimulation produces LB, a contraction of longitudinal muscles at the site of the touch and relaxation of longitudinal muscles in the same segment, but on the other side of the body. CI first appears at $\sim 55 \%$ ED and is elicited by touch for 1 or $2 \mathrm{~d}$, then wanes as LB appears (Reynolds et al., 1998b). The circuit that produces LB in the adult leech has been characterized in detail (Fig. $1 B, C$ ) previously (Kristan et al., 2000) and includes central inhibitory synaptic connections from inhibitory motor neurons [dorsal inhibitor cell 1 (DI-1) and ventral inhibitor cell 2 (VI-2)] onto excitatory motor neurons [dorsal exciter cell 3 (DE-3) and ventral exciter cell 4 (VE-4), respectively] (Fig. 1C). These inhibitory connections are GABAergic (Cline, 1986).

To test whether the switch from CI to LB might be attributable to the onset of GABAergic inhibition, we characterized the behavior of three groups of leeches: embryos that produced CI in response to touch (at $54-58 \% \mathrm{ED}$ ), embryos that produced adult-like LB in response to the same stimulation (at 60-65\% $\mathrm{ED})$, and juveniles ( $>100 \% \mathrm{ED})$. In all three groups, we tested the animals in either normal embryo water or saline (depending on whether they were studied intact or dissected), which was replaced by the same solution containing the GABA blocker BMI. In each solution, a selected segment in the mid-body was touched repeatedly using a calibrated amount of pressure, and the response was recorded and quantified. In embryos at 52-54\% ED, touching produced $\mathrm{CI}$ both in control solution and in BMI solution (Table 1, Fig. 2A). In embryos at 65\% ED (Fig. 2B), as well as in juveniles (Fig. $2 C, D$ ), touch typically elicited LB when an embryo was in control solution and CI when it was in BMI solution (Table 1). This effect of BMI was reversible; after washout, the behavior returned to LB (Fig. 2C,D). These results are consistent with our hypothesis that $\mathrm{CI}$ is $\mathrm{LB}$ without inhibitory chemical synaptic transmission and suggest that inhibition is well established by $65 \% \mathrm{ED}$.

\section{Voltage-sensitive dyes suggest the onset time of inhibitory connections}

To determine more directly when synaptic connections developed, we used voltage-sensitive dyes to record the activity of neurons with somata on the dorsal side of the ganglion, where most motor neurons are located. We used FRET dyes that provide single-cell resolution (Cacciatore et al., 1999) at 2-5 mV sensitivity (Taylor et al., 2003). We impaled and repeatedly depolarized at $1 \mathrm{~Hz}$ a dorsal inhibitory motor neuron, DI-1, while imaging

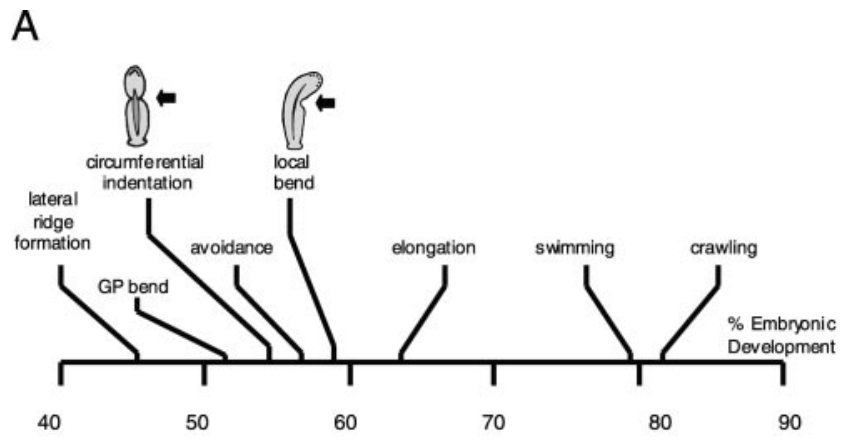

B

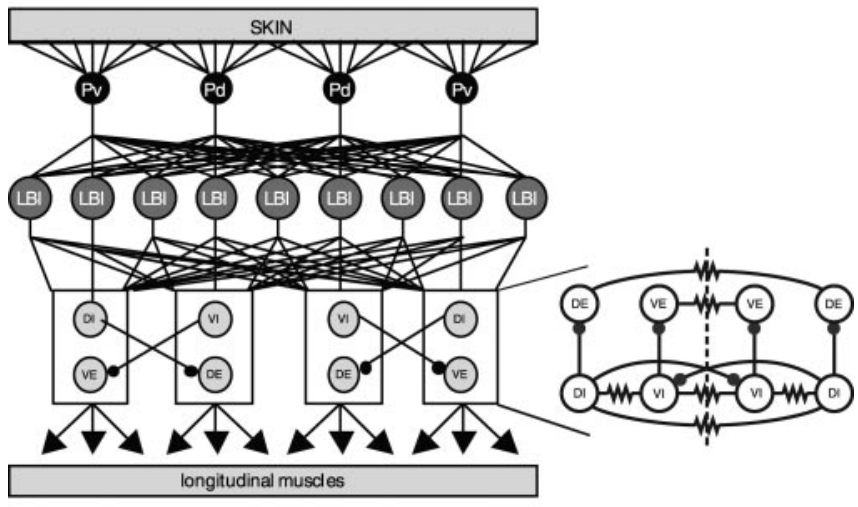

C

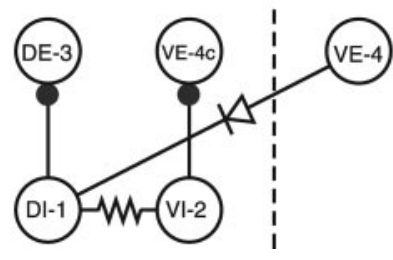

Figure 1. Development of behaviors and LB circuit of the leech. $\boldsymbol{A}$, Time line for development of behavior in Hirudo embryos. Time is measured as a percentage of total ED (\% ED). GP, Germinal plate. Drawings of $\mathrm{Cl}$ and LB illustrate the behaviors. Moderate pressure produces $\mathrm{Cl}$ between 55 and 60\% ED and LB after 60\% ED. Data are modified from Reynolds et al. (1998b). $\boldsymbol{B}$, Left, Diagram of the LB circuit drawn as though a leech was cut along the ventral midline and stretched out. The four P mechanosensory neurons that innervate the ventral (Pv) and dorsal $(\mathrm{Pd})$ regions of the body wall are shown in black, LBls are shown in dark gray, and inhibitory (DI and $\mathrm{VI}$ ) and excitatory (DE and VE) motor neurons innervating longitudinal muscle are shown in light gray. The only known inhibitory connections in the circuit (represented by black circles at the end of the lines connecting the neurons) are made between motor neurons. Data are adapted from Kristan et al. (2000). Right, Detailed longitudinal motor neuron circuit. Each segmental ganglion contains 16 bilateral pairs of longitudinal motor neurons interconnected centrally by both chemical (filled circles) and electrical (resistor symbol) synapses. The central connections for 12 of these pairs are summarized here. The dashed line indicates the ventral midline. Each circle represents a class of two to four neurons that form the same pattern of connections. Class abbreviations indicate the region of longitudinal muscle innervated (e.g., dorsal vs ventral) and the neuronal polarity (i.e., excitatory vs inhibitory). DI, Dorsal inhibitors; $\mathrm{DE}$, dorsal excitors; $\mathrm{VI}$, ventral inhibitors; $\mathrm{VE}$, ventral excitors. On each side of the ganglion, there are two DIs, four DEs, three VIs, and three VEs. All neurons within each class are electrically coupled; their connections have been left out for graphic clarity. C, Reduced longitudinal motor neuron circuit. Each circle represents an individual neuron from the classes shown in $\boldsymbol{B}$. Most leech motor neurons project contralaterally, whereas cell VE-4 projects ipsilaterally. Therefore, the soma of cell VE-4 is contralateral to the somata of other motor neurons that have the same motor fields, and we designate it VE-4c. Filled circle, Inhibitory chemical synapse; resistor, nonrectifying electrical synapse; diode, rectifying electrical synapse. 
$\sim 50$ additional neurons (Fig. 3). We used isolated ganglia from embryos at $58 \% \mathrm{ED}$ through adulthood. In all ganglia, some of the neurons showed membrane potential trajectories that oscillated at the same frequency as the stimulated neuron (Fig. $3 B$ ). The polar plot (Fig. 3C) shows the magnitudes and phases of the coherence for the optical signals of all neurons visible in the image. Neurons with significant coherence values (outside the dashed lines in Fig. 3C) have been colored in Fig. 3A-C. Brighter colors represent higher coherence values, and different hues represent the phase relationships of the responses relative to the stimulus. From these data, we infer that the in-phase neurons (those with small phase angles) (Fig. 3, green) were electrically coupled to the driven neuron, whereas the out-of-phase neurons (large phase angles) (Fig. 3, blue) were inhibited by the driven neuron. Additional experiments, described below, were consistent with this interpretation. This pattern is also seen in ganglia from adult leeches (Cacciatore et al., 1999).

The voltage-sensitive dye experiments indicated the connectivity within the population of motor neurons as a whole, so that we could see that the phasing of the coherent neurons varied over developmental time. At 58\% ED, before LB begins, all coherent neurons were nearly in-phase with the driven neuron (green), whereas neurons at $70 \% \mathrm{ED}$ or later fell into two categories: one group was in-phase with cell DI-1 (electrically coupled with DI-1) and the other group was out-of-phase (inhibited by DI-1). The locations of the neurons that were coherent with the stimulated cell DI-1 at 58\% ED (Fig. 4A), 70\% ED (Fig. 4C), and adults (Fig. 4B) were compared with the standard map of a leech mid-body ganglion. Despite some variability from ganglion to ganglion, several regularities appeared (Fig. 4D): (1) inphase coherence (green) developed before out-of phase coherence (blue); (2) the number of neurons inphase with cell DI-1 remained constant through development (one-way ANOVA; $F_{(2,10)}=0.41$; NS), whereas the number of out-of-phase neurons increased (one-way ANOVA; $\left.F_{(2,12)}=37.9 ; p<0.001\right)$; and (3) some neurons retained their phasing (e.g., the neurons near DI-1 remained in-phase), whereas others changed their phasing (e.g., contralateral cells 2, 101 , and 102). During this period, some new in-phase neurons appeared, keeping the number of in-phase neurons constant although their identity changed. These results indicate that electrical coupling was present before LB behavior began and did not change significantly during further development. In contrast, inhibition came on later, appearing initially during the transition from CI to LB. Similar results were obtained when a ventral inhibitory motor neuron, VI-2, was chosen as the cell to be stimulated (data not shown).
B

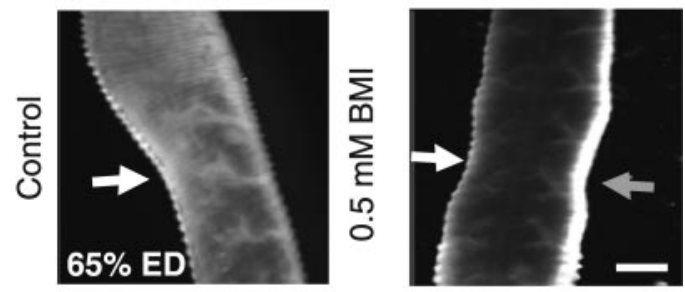

D

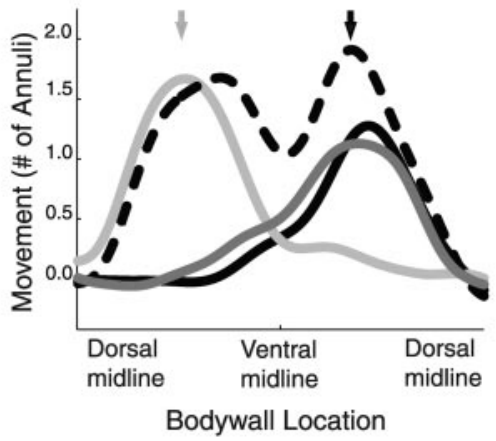

Bodywall Location

Figure 2. Switching from $\mathrm{Cl}$ to $\mathrm{LB}$ depends on the formation of GABAergic connections. $\boldsymbol{A}$, Cl executed by a $51 \%$ ED embryo in response to mechanical stimulation. The white arrow indicates the location of the touch, and the gray arrow shows contraction contralateral to the touch. At this stage, touch elicited Cl both in control saline solution and in a solution containing $0.5 \mathrm{~mm} B M I$. B, A 65\% ED embryo in which mechanical stimulation of a segment (white arrow shows the location) elicited LB in a contro (left) and Cl in a solution containing $0.5 \mathrm{~mm} \mathrm{BMI} \mathrm{(right).} \mathrm{C,} \mathrm{A} \mathrm{100 \%} \mathrm{ED} \mathrm{juvenile.} \mathrm{In} \mathrm{this} \mathrm{embryo,} \mathrm{the} \mathrm{ganglion} \mathrm{in} \mathrm{the}$ stimulated segment was exposed and the body wall was pinned out. Mechanical stimulation of the segment (white arrow) elicited LB in 列 ysis of images from dissected juvenile leech. The solid curves show the strength and location of longitudinal contraction in norma midline. The dotted line shows the response to a touch on the right side in $0.5 \mathrm{~mm}$ BMI dissolved in normal saline. The medium gray line shows the response to touch on the right after 20 min of washing with normal saline.

Electrophysiological tests pinpoint the onset time of electrical connections among motor neurons

Recording with voltage-sensitive dyes told us which neurons were in-phase with the driven neuron but could not clearly distinguish excitatory chemical synaptic transmission from electrical junctions. To further explore whether the motor neurons establish their electrical connections first and only later form chemical synapses, we recorded from pairs of motor neurons electrophysiologically. We focused on four interconnected motor neurons (Fig. 1C) (DI-1, VI-2, DE-3, and VE-4) because each of them can be identified uniquely by morphological criteria, and they represent the fours types of motor neurons in the circuit that produces LB. We recorded from 232 pairs of neurons in isolated embryonic ganglia ranging from 51 to $85 \%$ ED. In each case, after making the recordings, we identified the neurons morphologically. Data were rejected if we could not positively identify both neurons of the pair. 
A
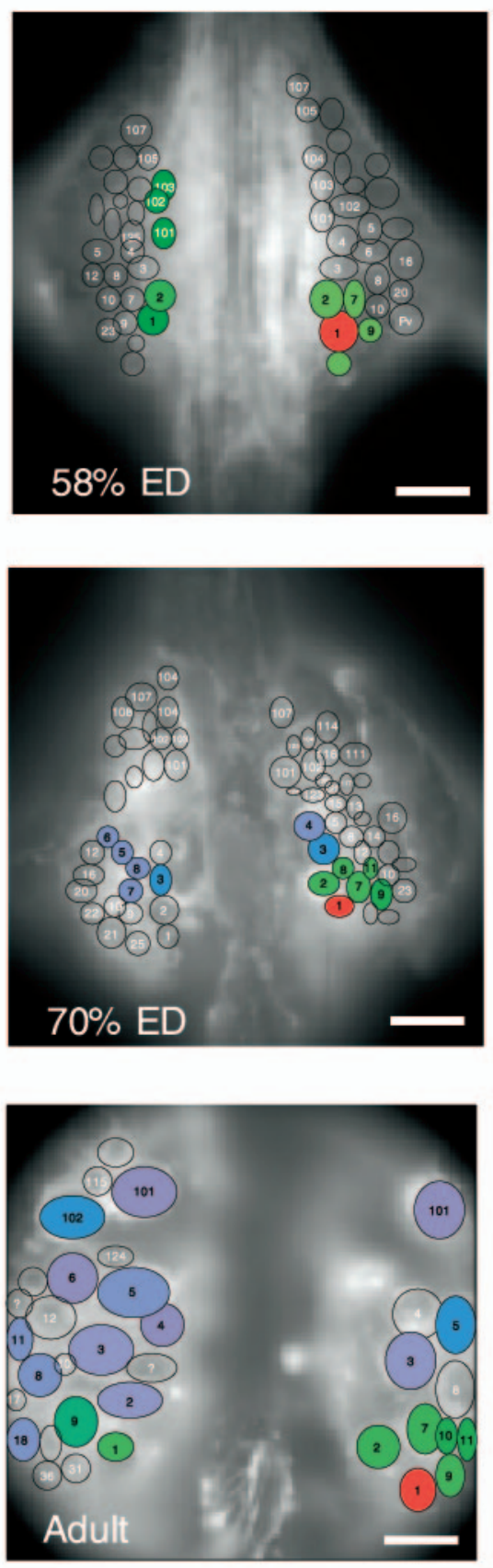

B

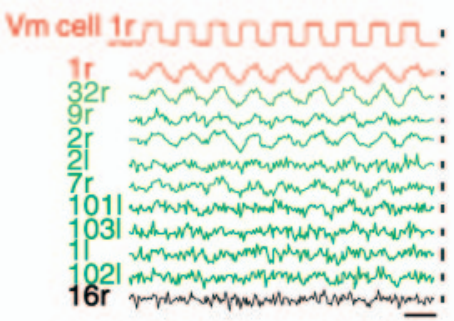

scale bars: $20 \mathrm{mV}, 0.2 \%, 1 \mathrm{sec}$
C
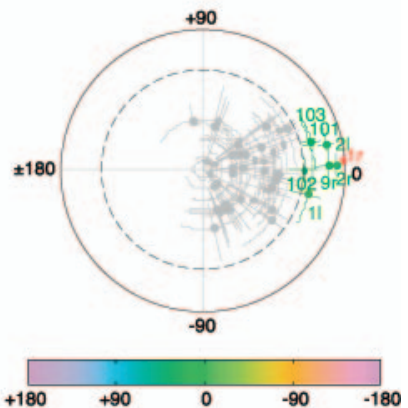

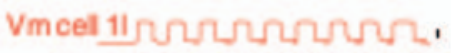

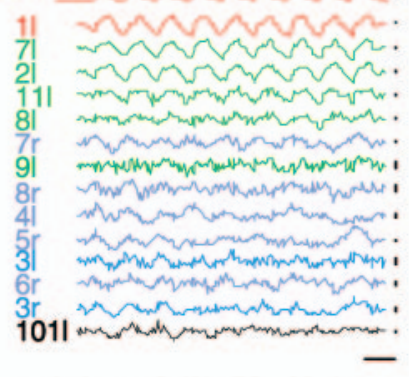

scale bars: $0.1 \%, 50 \mathrm{mV}, 1 \mathrm{sec}$
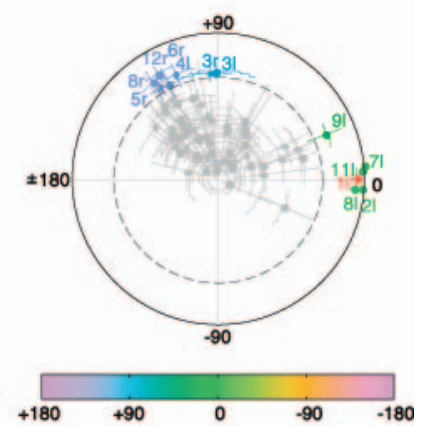

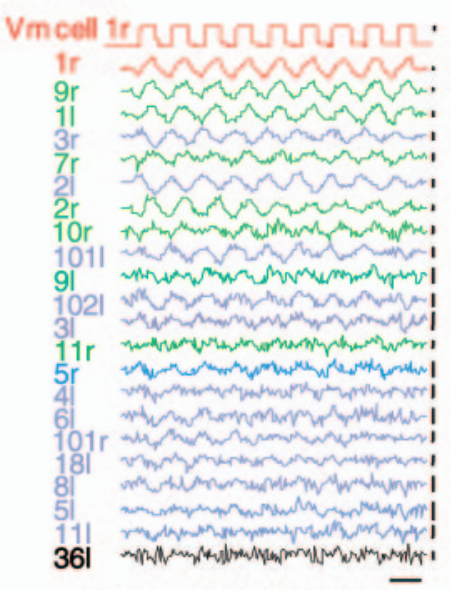

scale bars: $0.1 \%, 50 \mathrm{mV}, 1 \mathrm{sec}$

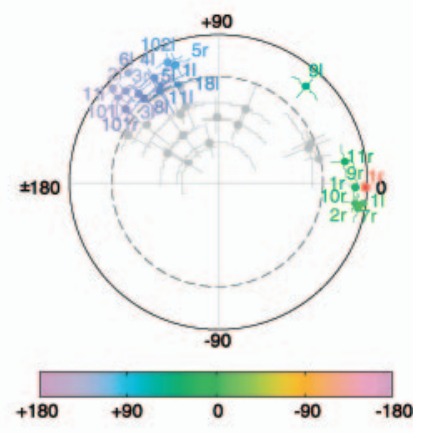

Figure 3. FRET voltage-sensitive dye recordings of motor neuron activity revealed when inhibition appeared during development. $A$, Images of the dorsal surface of a mid-body ganglion used to record neuronal activity with voltage-sensitive dyes. Hand-drawn ellipses indicate the boundaries of neuronal somata. A motor neuron, DI-1, was impaled and depolarized (shown in red); neurons that were significantly coherent with the driven cell are shown in green (indicating that they were in-phase with the driven cell) or blue (indicating that they were out-of-phase with the driven cell). The results from three developmental stages are shown. Scale bars, $50 \mu \mathrm{m}$. $\boldsymbol{B}$, Records of the electrical and fluorescence signals from the most coherent neurons shown in $\boldsymbol{A}$. The colors correspond to phasing of the responses: records shown in green were near $0^{\circ}$ phase relative to the stimulated cell (i.e., they depolarized when the driven cell was depolarized), and those shown in blue were at $90-150^{\circ}$ (they hyperpolarized when the driven cell was depolarized). The records shown in black indicate noncoherent neurons. Calibration bars are displayed below and to the right of the records. The signals are lined up in decreasing order of their coherence values, with the maximum value at the top. In all cases, stimuli were depolarizations lasting $500 \mathrm{~ms}$ and delivered at $1 \mathrm{~Hz}$ at an intensity that varied with stage: $0.3 \mathrm{nA}$ at $58 \% \mathrm{ED}, 0.8 \mathrm{nA}$ at $70 \% \mathrm{ED}$, and $2 \mathrm{nA}$ in the adult. C, Polar plots show the coherence phase (the angle from $0^{\circ}$ ) and magnitude (distance from the center of the plot) of the responses of all neurons observed in the image. Values greater than the dashed line are coherent, at the $95 \%$ confidence limit, with the stimulus.

Embryonic neurons had resting membrane potentials $\left(V_{\text {rest }}\right)$ of -10 to $-30 \mathrm{mV}$, lower than the $V_{\text {rest }}$ of -40 to $-50 \mathrm{mV}$ seen in adults. We believe these relatively low values of $V_{\text {rest }}$ are attributable to immaturity. However, the low values of $V_{\text {rest }}$ did not preclude electrical activity. We observed both action potentials and IPSPs in embryonic neurons with $V_{\text {rest }}$ values as low as -10 $\mathrm{mV}$. We recorded action potentials from neurons as early as $51 \%$ $\mathrm{ED}$, but not earlier. At this early stage, neurons generated only 
short bursts of action potentials either when they were impaled or at the offset of a hyperpolarizing current pulse and then would remain silent. Individual action potentials recorded in the soma were similar in size $(<5 \mathrm{mV})$ and appearance to those seen in adult motor neurons (Stuart, 1970; Ort et al., 1974). By 55\% ED, some of the neurons generated sustained trains of action potentials in response to prolonged depolarization, just as they do in adults, and most of them generated such prolonged trains in embryos at $\geq 62 \%$ ED.

\section{Electrical synapses that last into adulthood}

In adult leeches, cell DI-1 is electrically coupled with VI-2 and with VE-4 (Fig. 1C). To investigate the development of these electrical connections, we recorded simultaneously from either DI-1 and VE-4 $(n=35)$ or DI- 1 and VI-2 $(n=23)$ in embryos from 51 to $85 \%$ ED. For both DI- 1 to VE-4 (Fig. 5A) and DI-1 to VI-2 (data not shown), we observed electrical connections in embryos as young as $51 \%$ ED. The coupling strength was lowest in the youngest embryos and increased throughout development until it reached a maximum at $75 \% \mathrm{ED}$, when it was significantly greater than the adult coupling strength (Fig. 5B). This drop in coupling strength between $75 \%$ ED and adulthood could be caused either by a decrease in the junctional conductance (Bennett, 1966) or by a decrease in the input resistance of the target cell. Because the input resistance did not change significantly between $65-85 \%$ ED and adulthood ( $p=0.92$ ) (Fig. $5 E$ ), we conclude that the junctional conductance drops. To test this conclusion, we estimated the junctional conductance from our measured values of coupling strength and input resistance (Bennett, 1966). The DI-1 to VE-4 electrical synapse had a mean junctional conductance of $6.1 \mathrm{nS}$ from $51-65 \% \mathrm{ED}$ and $5.7 \mathrm{nS}$ in adults. In contrast, there was no significant change in coupling strength over the same period for the DI-1 to VI-2 electrical synapse $(p=0.62)$.

\section{Transient electrical synapses}

As suggested in the experiments with voltage-sensitive dyes (Fig. $4 A$ ), some pairs of neurons had electrical coupling in early embryos that did not persist into adults. For example, cells DI-1 and DE-3 are not electrically coupled in adults; instead, cell DI-1 makes a chemical inhibitory synapse onto cell DE-3. In embryos younger than $55 \% \mathrm{ED}$, a step of depolarizing current into DI-1 elicited depolarization in DE-3 (Fig. 5C). Because GABA can initially be excitatory in developing nervous systems, only later becoming inhibitory as the target neurons mature (Ben-Ari, 2002), this depolarization might have indicated an excitatory chemical synapse from cell DI-1 onto cell DI-3. Recording from cell DI-1 while driving cell DE-3 and injecting ramp stimuli, however, confirmed the electrical nature of the synapse. This electrical synapse was doubly rectifying, as shown by passing a current ramp first into DI-1 and then into DE-3 (Fig. 5C): in both directions, hy-
B

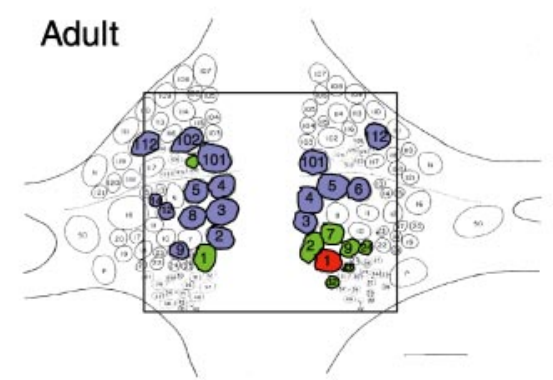

D

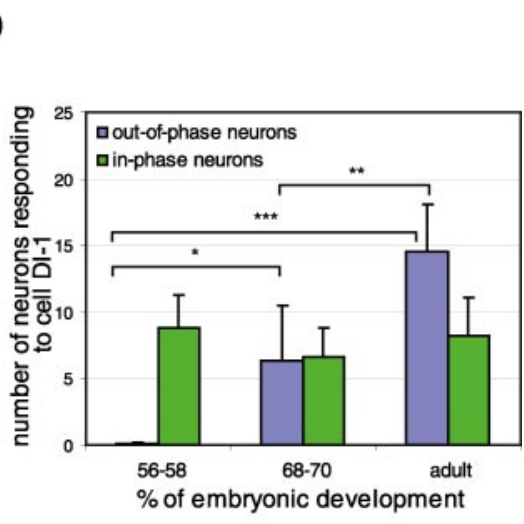

phase with the driven neuron

Follower cells that were out of phase with the driven neuron sexperiments. $\boldsymbol{A}-\boldsymbol{C}$, Map of a mid-body ganglion (dorsal side) indicating the position of neurons found to be coherently either in-phase (green) or out-of-phase (blue) with the driven cell DI-1 (red) at 58\% ED (A), 70\% ED (C), and in adults $(\boldsymbol{B})$. The square on each ganglion indicates the area used in the experiments. Somata that were coherent in at least two experiments are colored: green if they were in-phase and blue if they were out-of-phase neurons that were in-phase with the driven neuron, and blue bars show neurons that were out-of-phase with the driven neuron ( $n=3-6$ animals for each stage; values are mean \pm SEM; ANOVA; $\left.{ }^{*} p<0.05 ;{ }^{* *} p<0.01 ;{ }^{* * *} p<0.001\right)$.

perpolarization passed more readily than depolarization. We found a relatively small number of such transient electrical synapses (4 of 38). We saw no instances of "inappropriate" electrical coupling (i.e., coupling between cells that is not seen in adults) in embryos older than $65 \%$ ED.

On two occasions, we saw a mixed chemical and electrical synapse between DI-1 and DE-3 (Fig. 5C, 60\% ED). In these cases, stimulating DI-1 with a current step generated a small IPSP in DE-3, but applying a ramp stimulus to DE-3 gave a ramp response in DI-1, demonstrating that the cells were electrically coupled. Stimulating DI-1 with a current ramp gave a mixed chemical-electrical response in DE-3 (data not shown). The coupling strength between DI-1 and DE-3 was highest at the younger stages of development (Fig. 5D) and decreased as development progressed. The high coupling strength observed between DI-1 and DE-3 in the 51-65\% ED embryos resulted from a high junctional conductance. In 51-65\% ED neurons, the mean junctional conductance of the DI-1 to DE-3 electrical synapse was 800 pS, which was nearly five times the value of $168 \mathrm{pS}$ in adults.

We tested whether transient electrical synapses formed only between cells that would later connect by chemical synapses. We found evidence for one electrical connection between motor neurons that are never connected in adults, between VI-2 and DE-3. Although this incidence was low (4 of 30), it is similar to the probability of transient electrical connections between DI-1 and DE-3 (4 of 38) and between VI-2 and VE-4c (2 of 28), pairs that are connected in adults. We never found electrical coupling be- 

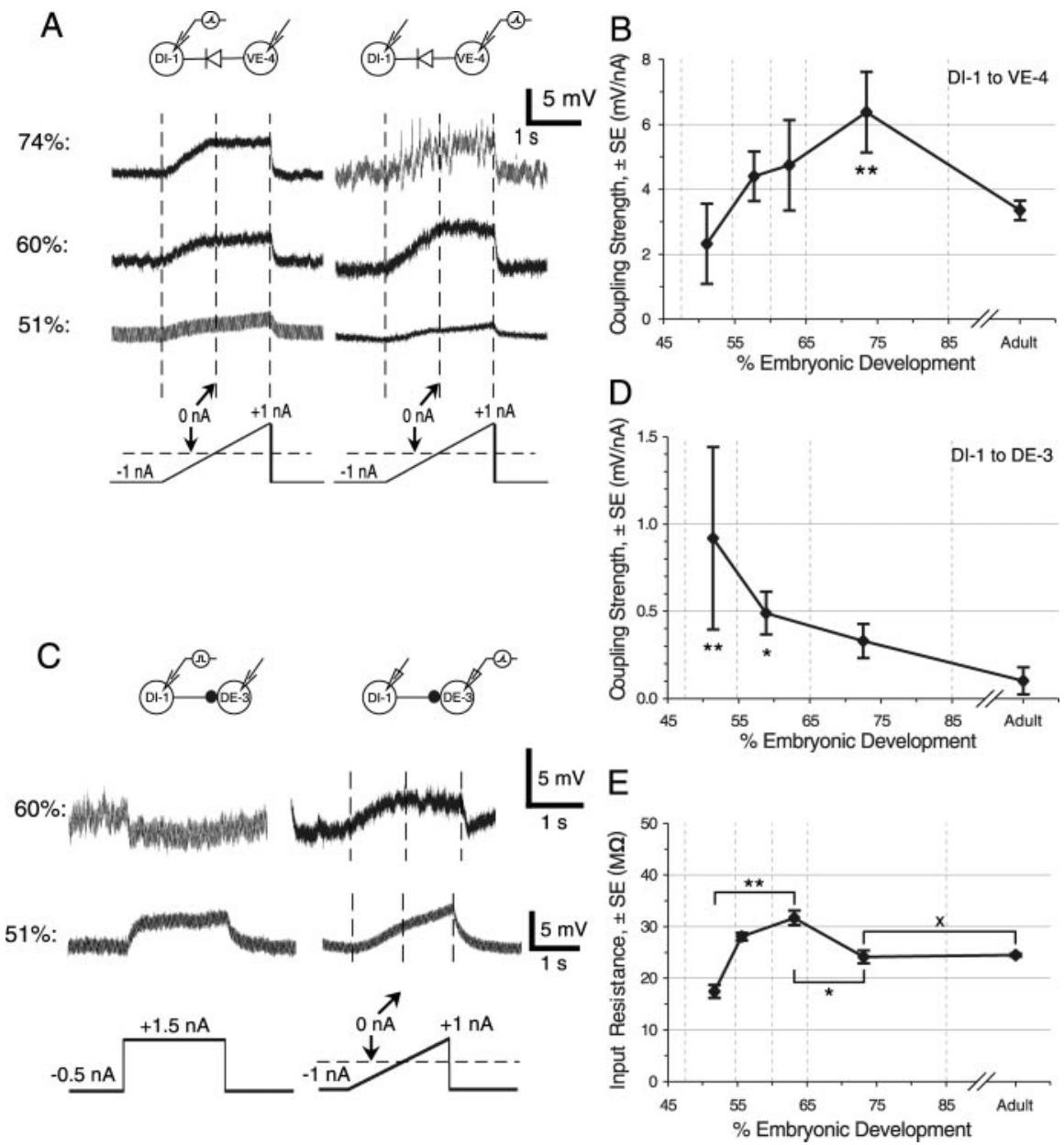

Figure 5. Development of electrical synapses. $A$, DI-1 to VE-4. Stimulating DI-1 while recording from VE-4 (left) or stimulating VE-4 while recording from DI-1 (right) revealed that electrical synapses were present as early as 51\% ED. Coupling strengths increased as development progressed. Electrical synapses in embryos commonly exhibited "negative rectification" for both directions of current flow (double rectification), shown by the larger responses during the negative portion of the stimulus ramp. $\boldsymbol{B}$, The coupling strength for the DI-1 to VE-4 electrical synapse increased during development up to 85\% ED and was significantly greater at $65-85 \%$ ED than in adults. Data from individual neurons were binned by developmental stage. The first bin was placed so that it included all data from embryos younger than the earliest stage at which chemical synapses were detected ( $\sim 55 \% \mathrm{ED})$; the other bin boundaries were placed at convenient intervals. The asterisks indicate values significantly different from adults $\left(t\right.$ test; ${ }^{*} p<$ $0.05 ;{ }^{* *} p<0.01$ ). In this graph and all similar ones, vertical dashed lines mark the bin boundaries, and the $x$-axis position of each point indicates the mean stage for all of the data points within that bin. Values are mean \pm SEM. The asterisks indicate points at which the coupling strength was significantly different from the adult value. $n=5,13,10,8$, and 14 from left to right. The coefficient of variation (CV) was $82.2 \%$ in $51-65 \%$ ED embryos and 33.0\% in adults. C, Transient electrical coupling between DI-1 and DE-3. Paired recordings from DI- 1 and DE- 3 at $51 \% \mathrm{ED}$ and at $60 \% \mathrm{ED}$ are shown. Stimulating $\mathrm{DI}-1$ with a positive current pulse (left) generated a small IPSP in DE-3 at 60\% ED but a depolarization at 51\% ED. Stimulating either DE-3 or DI- 1 (data not shown) with a ramp (right) generated a ramp response in the postsynaptic cell, confirming that the two cells were electrically coupled. $\boldsymbol{D}_{\text {, }}$ Coupling strength for the DI- 1 to DE-3 electrical synapse. The asterisks indicate points that are significantly different from adults. $n=6,32,16$, and 27 from left to right. Because of the transient nature of the synapse, the CV at $51-65 \% \mathrm{ED}$ was very high (144.9\%). $\boldsymbol{E}$, Changes in input resistance during development. Embryonic input resistances have been normalized to compensate for the effects of high-resistance electrodes (see Materials and Methods). $n=19,43,18,56$, and 23 from left to right.

tween VI-2 and DE-3 after 65\% ED (0 of 10 in adults). We recorded from several pairs of motor neurons that were never coupled. For example, DE-3 and VE-4, excitatory motor neurons to dorsal and ventral longitudinal muscles, are never connected, either electrically or chemically, in the adult nervous system ( 0 of 4) (Ort et al., 1974; Granzow and Kristan, 1986), and we never observed connections between these cells in embryos (0 of 19). We conclude, therefore, that electrical connections among motor neurons are neither completely promiscuous and random nor are they limited entirely to pairs of cells that later make chemical synapses.

\section{Development of chemical synapses}

The behavioral and voltage-sensitive dye experiments suggested that inhibitory synapses among motor neurons can first be recorded in embryos when they begin to produce LB. Paired intracellular recordings from DI- 1 and DE-3 $(n=44)$ or from VI- 2 and VE-4c $(n=32)$ in isolated ganglia from embryos at 51-74\% ED were consistent with this result. At 51\% ED, an electrical connection was apparent (Fig. $6 A)$. Then, the earliest stage at which we observed inhibitory responses was 55\% ED (Fig. 6A), in both DI- 1 to DE-3 and VI-2 to VE-4c. At this stage, the IPSPs were small (Fig. $6 B$ ) and decremented rapidly (Fig. 6A). From $60-74 \%$ ED, the IPSP size for both synapses increased significantly. The IPSP size increased further before adulthood $(p<0.01)$. An additional metric for measuring synapse formation is their probability of detection. For this, we counted as IPSPs any response $-1 \mathrm{mV}$ or greater in the postsynaptic cell after stimulation of the presynaptic cell. (The value of $-1 \mathrm{mV}$ was chosen somewhat arbitrarily; we obtained similar results when we set the threshold at different values.) Before $55 \%$ ED, stimulating DI-1 produced no detectable IPSPs in DE-3; stimulating VI-2 produced no response in VE-4c (Fig. 6C). The probability of eliciting an IPSP then increased steadily to nearly 0.8 in embryos at $60-65 \%$ ED. This value was significantly greater than the probability of an IPSP at $51-60 \%$ ED (Fisher's exact test; $p<0.05)$ but not significantly different from the probability at $65-74 \%$ ED. Thus, the probability of eliciting an IPSP reached its maximal value during the same developmental period when the IPSP reached its maximal embryonic amplitude.

One factor contributing to the small size of early embryonic IPSPs may have been their short duration (Fig. 6A, 55\% ED). This decrement was still observable at $74 \% \mathrm{ED}$, when the sustained IPSP size for DI- 1 to DE-3 (i.e., the size near the end of a pulse) was only $72.7 \pm 7.5 \%$ of its peak size $(n=10)$ and significantly less than the sustained amplitude/peak amplitude of $99.2 \pm$ $0.2 \%(n=23)$ seen in adults $(p<0.01)$. Interestingly, this decrement in the IPSPs of embryonic neurons appeared to have no effect on the ability of embryos to execute the LB behavior.

\section{Discussion}

Timing of synapse formation

We studied the development of connections between identified motor neurons that form a circuit generating the local bend behavior in the medicinal leech. Our results revealed that the development of this circuit occurs in three stages: (1) electrical synapses form first (at $~ 51 \%$ ED); (2) chemical synapses appear $\sim 1$ 


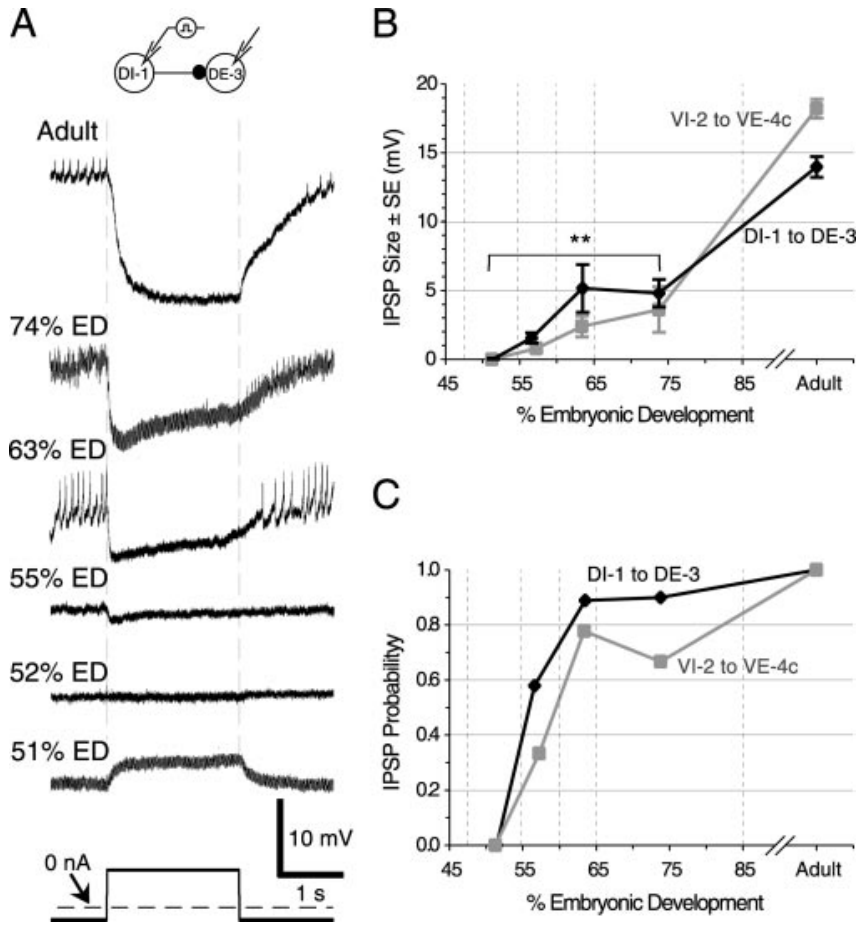

Figure 6. Development of chemical synapses. $\boldsymbol{A}$, Examples of intracellular recordings made at different developmental stages of activity in DE-3 in response to stimulating DI- 1 with current pulses of 2- $4 \mathrm{nA}$. (Because experiments are terminal, each recording is from a different embryo.) B, IPSP size for the DI- 1 to DE-3 and VI-2 to VE-4c synapses. IPSP size was measured at the maximal amplitude of the hyperpolarization. IPSP size for both synapses was zero before $55 \%$ $\mathrm{ED}$ and gradually increased to $\sim 5 \mathrm{mV}$ by $74 \% \mathrm{ED}$. This amplitude was still significantly smaller than the IPSP size of $10-20 \mathrm{mV}$ regularly seen in adults. Values are mean \pm SEM. The asterisks indicate values significantly different from adults $\left(t\right.$ test; $\left.{ }^{* *} p<0.01\right)$. C, Probability of IPSPS exceeding a threshold of $1 \mathrm{mV}$ for the DI- 1 to DE-3 and VI-2 to VE-4c synapses. No cell pairs had IPSPs $>1 \mathrm{mV}$ before $55 \% \mathrm{ED}$. In $\boldsymbol{B}$ and $\boldsymbol{C}, n=6,19,9,10$, and 23 from left to right for DI- 1 to DE- 3 and $n=2,15,9,6$, and 10 for VI-2 to VE-4C.

$\mathrm{d}$ later (55\% ED) and reach a plateau in size between 60 and $65 \%$ $\mathrm{ED}$; and (3) transient (inappropriate) electrical connections disappear between $62-65 \%$ ED. Figure $7 A$ summarizes these results.

\section{Development of behavior}

A previous behavioral study reported that LB first appears at $\sim 59 \%$ ED and is preceded by CI, an embryonic behavior (Reynolds et al., 1998b). We found that the switch from CI to LB takes place when some electrical synapses that have been established between motor neurons are replaced by inhibitory chemical synapses. Comparing the timing of these synaptic events with the timing at which first CI and then LB appear (Fig. 7B) suggests that $\mathrm{CI}$ is produced by electrical connections exclusively and that the nature of the connectivity among them changes to generate LB. The probability of eliciting CI increases at the time when electrical connections among motor neurons become stronger, whereas LB first appears and then becomes more likely as the inhibitory chemical synaptic connections between motor neurons appear and grow stronger. This proposed causal relationship is reinforced by treating embryonic and juvenile leeches with bicuculline, an inhibitor of GABAergic synaptic transmission. During treatment with the drug, $C I$ is unaffected in young embryos, but $\mathrm{LB}$ reverts to $\mathrm{CI}$ in older leeches and juveniles (Fig. 2).

A similar developmental progression is seen in the vertebrate spinal cord, in which locomotor programs appear during embryogenesis before they are of any obvious functional use (Bate,

\section{A. Behavioral and synaptic development}

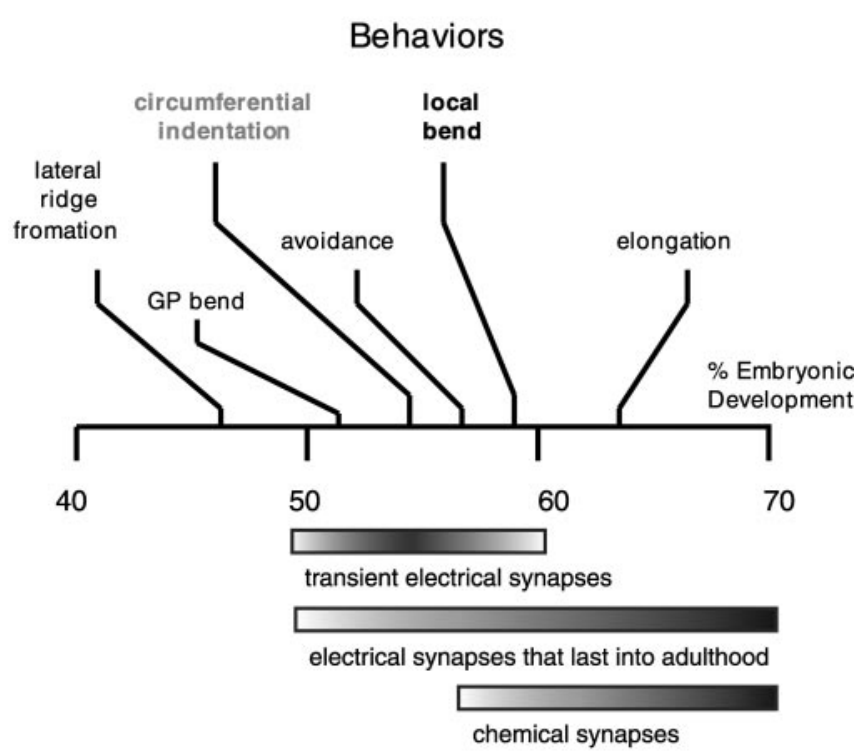

\section{B. Ontogeny of local bending}

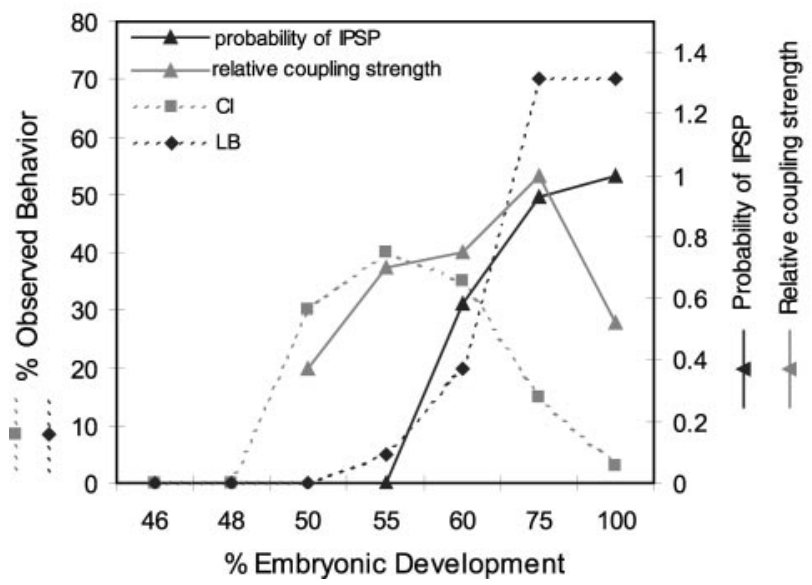

Figure 7. A, Timing of synaptic development relative to the onset of embryonic and adult behaviors [adapted from Reynolds et al. (1998b)]. Motor neurons were electrically active and formed both stable and transient electrical synapses by $51 \%$ ED. Inhibitory chemical synapses first appeared at 55\% ED and reached their maximum embryonic amplitude between 60 and $65 \% \mathrm{ED}$, which is close to the stage when $\mathrm{LB}$ appears. Transient electrical synapses faded as chemical synapses matured, and they were no longer detected after $65 \%$ ED. GP, Germinal plate. $\boldsymbol{B}$, Comparing electrical and chemical connectivity with the development of LB. Solid lines show the coupling strength, expressed as a percentage of its maximum value (representing the electrical synapses) or probability (representing chemical synapses) of IPSP. The dashed lines show the percentage of individuals displaying each behavior as development proceeded. The behavioral data are smoothed from Reynolds et al. (1998b).

1999). In at least some of these circuits, the first connections are electrical and include connections among the motor neurons themselves (Chang et al., 1999; Tresch and Kiehn, 2000). These electrical connections produce oscillatory motor patterns for a time, although their function is ultimately taken over by chemical synapses (O'Donovan et al., 1992; Sillar and Simmers, 1994; Cazalets et al., 1996; Brocard et al., 1999; Nishimaru and Kudo, 2000).

A different kind of developmental progression is seen in the 
pattern of side-to-side coordination of motor output from the developing vertebrate spinal cord. Initially GABAergic synapses produce an excitatory effect on target cells and generate simultaneous rhythmic activity in both the left and the right sides of the cord. Later, because of maturational changes in the postsynaptic neurons, GABA becomes inhibitory, which causes output to the left and right sides to became alternating (Nakayama et al., 2002). Eventually, glycine receptors take over this inhibitory role (Nishimaru and Kudo, 2000; Ben-Ari, 2002; Nakayama et al., 2002).

In our experiments, bicuculline produced no effect on CI, and we conclude that the circuit that produces $\mathrm{CI}$ does not depend on GABAergic excitatory transmission. Instead, the circuit is connected by means of electrical synapses that set up a scaffold on which many appropriate chemical connections ultimately form.

Based on several different experimental approaches, we conclude that physiologically active electrical connections are present in this circuit before LB appears and that some of them remain into adulthood but that inhibition among motor neurons is necessary to produce mature LB behavior. Our ability to identify individual motor neurons both for electrophysiological recording and by using the voltage-sensitive dyes to study their activity throughout the development of the leech CNS has shown that embryonic neurons establish connections quite specifically. The variability we saw was likely attributable to the transient nature of some of the connections.

Because the shift from CI to LB takes place when a leech embryo would normally be packed into its cocoon along with its siblings, there is little reason to think that the behaviors themselves are significant. We believe instead that the behaviors signal important changes in connectivity on the way to assembling a nervous system that is capable of producing adaptive behavioral responses to environmental stimuli.

\section{Transient electrical connections}

In embryos, we found transient electrical connections both between neurons that would be connected by a chemical synapse in adults (cells DI-1 to DE-3 and VI-2 to VE-4c) and between neurons that would not be connected at all in adults (cells VI-2 to DE-3). Thus, it appears that the embryonic electrical connections precede later chemical synapses between neurons of different types but not between neurons of the same type (e.g., the excitatory motor neurons DE-3 and VE-4). This pattern of early electrical coupling is unlike the one typically seen in vertebrates, in which neurons of the same cell type have been seen to couple with one another, both in the cortex (Kandler and Katz, 1995) and among ganglion cells in the retina (Penn et al., 1994).

Transient electrical connections among leech motor neurons were observed infrequently, however, occurring in only $\sim 10 \%$ of the recordings made from embryos at 51-65\% ED and never thereafter (Fig. 5). One possible explanation for this low incidence of transient coupling is that we simply misidentified neurons and were, in actuality, recording from pairs of neurons that we came to expect would be coupled. We consider this unlikely because (1) we always relied on critical morphological criteria for identification; (2) we sometimes observed mixed chemical and electrical synapses in embryos, a condition never seen in adults; and (3) injections of the low-molecular weight tracer Neurobiotin showed that the same pairs of motor neurons that we saw to be transiently electrically coupled were also dye-coupled at about the same frequency during these embryonic stages (Eisenhart and Kristan, 1999).

Transient electrical coupling has been reported for many regions of early development in the vertebrate nervous system
(Walton and Navarrete, 1991; Peinado et al., 1993b; Penn et al., 1994). In both leeches and vertebrates, transient electrical synapses commonly form before chemical synapses, with electrical uncoupling occurring at about the same time as the onset of chemical synaptic activity. This temporal correlation has often been taken as evidence for a causal relationship between the two events (Kandler and Katz, 1995). The timing of the events in the leech is consistent with this possibility, but there are clearly other potential functions for electrical junctions as well.

Studies of neuromuscular junctions in primary culture (Fischbach, 1972) suggest that transient electrical coupling might be a necessary step in the developmental program for making a chemical synapse. Recent studies of regenerating synapses between neurons in Helisoma showed that during regeneration, electrical connections are formed first and are replaced after a few days by chemical synapses (Szabo et al., 2004). In embryonic leeches, we found transient electrical synapses between cells that later would be connected via chemical synapses (DI-1 to DE-3 and VI-2 to VE-4c), but we also found electrical coupling between VI-2 and DE-3, two neurons that have no synaptic connection in the adult nervous system. Thus, transient electrical coupling may be a necessary step in the formation of a chemical synapse in leech, but it is not sufficient to ensure that a chemical synapse is formed.

\section{Specificity of chemical synapses}

In both the voltage-sensitive dye experiments and the electrophysiological studies, we never found evidence of transient chemical connections. We regard this result with caution, however, because such connections might occur at a frequency too low for us to detect, they might be so short-lived that we missed them, or they might be restricted to a narrow range of developmental stages that we never recorded from. Specificity of developing synapses has also been investigated in the cercal system of the embryonic cockroach (Blagburn et al., 1996), where the L sensory afferent forms a chemical synapse with the appropriate GI3 giant interneuron but not with the inappropriate GI2. Rearrangements of chemical synaptic connections are seen in the post-ED of the cricket cercal system (Shepherd and Murphey, 1986; Chiba et al., 1988) and between stages during metamorphosis in the moth (Levine and Truman, 1982; Jacobs and Weeks, 1990). These changes are likely to be related to functional changes in the body size and type of the animal, and, at least in the cricket, they do not depend on activity (Chiba and Murphey, 1991).

The neurons of invertebrates have been thought to make specific connections, regardless of surrounding events in the nervous system (Easter et al., 1985), whereas, in contrast, neurons in vertebrates are thought to project broadly and to establish diffuse synaptic circuits that are then stabilized or pruned in an activitydependent manner (Purves and Lichtman, 1980; Katz and Shatz, 1996; Ruthazer et al., 2003). This pattern has been observed in a variety of different locations: in the formation of the retinocollicular map (Debski and Cline, 2002; Ruthazer et al., 2003), during the construction of the neuromuscular junction (Lichtman and Colman, 2000), and in the organization of the mossy fiber projection in the cerebellum (Crespel et al., 1976). However, a different rule is followed in the development of intracortical circuits, in which considerable specificity has been observed even early in the developmental process (Katz, 1991; Borrell and Callaway, 2002; Bureau et al., 2004). The development of intracortical circuits may then be more similar to events in an invertebrate. In addition, it may be that motor neurons make much more specific connections from the start than do sensory neurons 
(Glover, 2000). However, in the vertebrates, it has thus far been possible only to follow the development of populations of neurons. Our ability to follow the development of identified neurons as they form synaptic connections has revealed that electrical connectivity established in the early development of the leech motor circuit appears to be very specific: connections are established between specific neurons, and some of them last into adulthood. However, intracellular recordings revealed that a low percentage of neurons formed transient electrical connections that disappeared as chemical synapses become established, and some neurons were transiently electrically coupled in connections that were broken by adulthood. It is thus possible that embryonic electrical connections serve multiple functions, from prefiguring behavioral circuits (Drapeau et al., 2002; Tresch and Kiehn, 2000) to pathfinding (Goodman and Shatz, 1993). The recent discovery of leech innexins in neurons (Dykes et al., 2004) presents the possibility of manipulating the expression of electrical junctions between these neurons to determine directly what their function is.

\section{References}

Bate M (1999) Development of motor behaviour. Curr Opin Neurobiol 9:670-675.

Ben-Ari Y (2002) Excitatory actions of GABA during development: the nature of the nurture. Nat Rev Neurosci 3:728-739.

Bennett MV (1966) Physiology of electrotonic junctions. Ann NY Acad Sci 137:509-539.

Bennett MVL (2000a) Electrical synapses, a personal perspective (or history). Brain Res Rev 32:16-28.

Bennett MVL (2000b) Seeing is relieving: electrical synapses between visualized neurons. Nat Neurosci 3:7-9.

Blagburn JM, Sosa MA, Blanco RE (1996) Specificity of identified central synapses in the embryonic cockroach-appropriate connections form before the onset of spontaneous afferent activity 373:511-528.

Bonnot AS, Whelan PJ, Mentis GZ, O’Donovan MJ (2002) Spatiotemporal pattern of motoneuron activation in the rostral lumbar and the sacral segments during locomotor-like activity in the neonatal mouse spinal cord. J Neurosci 22:RC203(1-6).

Borrell V, Callaway EM (2002) Reorganization of exuberant axonal arbors contributes to the development of laminar specificity in ferret visual cortex. J Neurosci 22:6682-6695.

Brocard F, Vinay L, Clarac F (1999) Development of hindlimb postural control during the first postnatal week in the rat. Dev Brain Res 117:81-89.

Bureau I, Shepherd GMG, Svoboda K (2004) Precise development of functional and anatomical columns in the neocortex. Neuron 42:789-801.

Cacciatore TW, Brodfuehrer PD, Gonzalez JE, Jiang T, Adams SR, Tsien RY, Kristan WB, Kleinfeld D (1999) Identification of neural circuits by imaging coherent electrical activity with FRET-based dyes. Neuron 23:449-459.

Cazalets JR, Borde M, Clarac F (1996) The synaptic drive from the spinal locomotor network to motoneurons in the newborn rat. J Neurosci 16:298-306.

Chang Q, Gonzalez M, Pinter MJ, Balice-Gordon RJ (1999) Gap junctional coupling and patterns of connexin expression among neonatal rat lumbar spinal motor neurons. J Neurosci 19:10813-10828.

Chiba A, Murphey RK (1991) Connectivity of identified central synapses in the cricket is normal following regeneration and blockade of presynaptic activity. J Neurobiol 22:130-142.

Chiba A, Shepherd D, Murphey RK (1988) Synaptic rearrangement during postembryonic development in the cricket 240:901-905.

Cline HT (1983) Tritium labeled gamma amino butyric-acid uptake selectively labels identifiable neurons in the leech central nervous system. J Comp Physiol 215:351-358.

Cline HT (1986) Evidence for GABA as a neurotransmitter in the leech. J Neurosci 6:2848-2856.

Crespel F, Mariani J, Delhaye-Bouchaud N (1976) Evidence for a multiple innervation of Purkinje cells by climbing fibers in the immature rat cerebellum. J Neurobiol 7:567-578.

Deans MR, Gibson JR, Sellitto C, Connors BW, Paul DL (2001) Synchronous activity of inhibitory networks in neocortex requires electrical synapses containing Connexin36. Neuron 31:477-485.
Debski EA, Cline HT (2002) Activity-dependent mapping in the retinotectal projection. Curr Opin Neurobiol 12:93-99.

Drapeau P, Saint-Amant L, Buss RR, Chong M, McDearmid JR, Brustein E (2002) Development of the locomotor network in zebrafish. Prog Neurobiol 68:85-111.

Dykes IM, Freeman FM, Bacon JP, Davies JA (2004) Molecular basis of gap junctional communication in the CNS of the leech Hirudo medicinalis. J Neurosci 24:886-894.

Easter Jr SS, Purves D, Rakic P, Spitzer NC (1985) The changing view of neural specificity. Science 230:507-511.

Eisenhart FJ, Kristan Jr WB (1999) A quantitative analysis of neurobiotin coupling demonstrates that leech neurons form transient electrical synapses during development. Soc Neurosci Abstr 25:202.11.

Fischbach GD (1972) Synapse formation between dissociated nerve and muscle cells in low density cell cultures. Dev Biol 28:407-429.

Galarreta W, Hestrin S (1999) A network of fast-spiking cells in the neocortex connected by electrical synapses. Nature 402:72-75.

Gibson JR, Beierlein M, Connors BW (1999) Two networks of electrically coupled inhibitory neurons in neocortex. Nature 402:75-79.

Glover JC (2000) Development of specific connectivity between premotor neurons and motoneurons in the brain stem and spinal cord. Physiol Rev 80:615-647.

Gonzalez JE, Tsien RY (1995) Voltage sensing by fluorescence resonance energy transfer in single cells. Biophys J 69:1272-1280.

Goodman CS, Shatz CJ (1993) Developmental mechanisms that generate precise patterns of neuronal connectivity. Cell 72:77-98.

Granzow B, Kristan Jr WB (1986) Inhibitory connections between motor neurons modify a centrally generated motor pattern in the leech nervous system. Brain Res 369:321-325.

Granzow B, Friesen W, Kristan Jr WB (1985) Physiological and morphological analysis of synaptic transmission between leech motor neurons. J Neurosci 8:2035-2050.

Jacobs G, Weeks J (1990) Postsynaptic changes at a sensory-to-motoneuron synapse contribute to the developmental loss of a reflex behavior during insect metamorphosis. J Neurosci 10:1341-1356.

Kandler K, Katz LC (1995) Neuronal coupling and uncoupling in the developing nervous system. Curr Opin Neurobiol 5:98-105.

Katz LC (1991) Specificity in the development of vertical connections in cat striate cortex. Eur J Neurosci 3:1-10.

Katz LC, Shatz CJ (1996) Synaptic activity and the construction of cortical circuits. Science 274:1133-1138.

Kristan Jr WB (1982) Sensory and motor neurons responsible for the local bending response in leeches. J Exp Biol 96:161-180.

Kristan Jr WB, Eisenhart FJ, Johnson LA, French KA (2000) Development of neuronal circuits and behaviors in the medicinal leech. Brain Res Bull 53:561-570.

Levine RB, Truman JW (1982) Metamorphosis of the insect nervous system changes in morphology and synaptic interactions of identified neurons. Nature 299:250-252.

Lewis JE, Kristan Jr WB (1998) A neuronal network for computing population vectors in the leech. Nature 391:76-79.

Lichtman JW, Colman H (2000) Synapse elimination and indelible memory. Neuron 25:269-278.

Lockery SR, Kristan Jr WB (1990) Distributed processing of sensory information in the leech. II. Identification of interneurons contributing to the local bending reflex. J Neurosci 10:1816-1829.

Long MA, Landisman CE, Connors BW (2004) Small clusters of electrically coupled neurons generate synchronous rhythms in the thalamic reticular nucleus. J Neurosci 24:341-349.

Lucas BD, Kanade T (1981) An iterative image registration technique with an application to stereo vision (IJCAI). In: Proceedings of the 7th International Joint Conference on Artificial Intelligence, pp 674-679. San Francisco: Morgan Kaufmann.

Marder E, Calabrese RL (1996) Principles of rhythmic motor pattern generation. Physiol Rev 76:687-717.

Muller KJ, Nicholls JG, Stent GS (1981) Neurobiology of the leech. Cold Spring Harbor, NY: Cold Spring Harbor Laboratory.

Nakayama K, Nishimaru H, Kudo N (2002) Basis of changes in left-right coordination of rhythmic motor activity during development in the rat spinal cord. J Neurosci 22:10388-10398.

Nishimaru H, Kudo N (2000) Formation of the central pattern generator for locomotion in the rat and mouse. Brain Res Bull 53:661-669. 
O’Donovan M, Sernagor E, Sholomenko G, Ho S, Antal M, Yee W (1992) Development of spinal motor networks in the chick embryo. J Exp Zool 261:261-273.

Ort CA, Kristan Jr WB, Stent GS (1974) Neuronal control of swimming in the medicinal leech. II. Identification and connections of motor neurons. J Comp Physiol 94:121-154.

Peinado A, Yuste R, Katz LC (1993a) Gap junctional communication and the development of local circuits in neocortex. Cereb Cortex 3:488-498.

Peinado A, Yuste R, Katz LC (1993b) Extensive dye coupling between rat neocortical neurons during the period of circuit formation. Neuron 10:103-114.

Penn AA, Wong ROL, Shatz CJ (1994) Neuronal coupling in the developing mammalian retina. J Neurosci 14:3805-3815.

Purves D, Lichtman JW (1980) Elimination of synapses in the developing nervous system. Science 210:153-157.

Reynolds SA, French KA, Baader A, Kristan Jr WB (1998a) Staging of middle and late embryonic development in the medicinal leech, Hirudo medicinalis. J Comp Neurol 402:155-167.

Reynolds SA, French KA, Baader A, Kristan Jr WB (1998b) Development of spontaneous and evoked behaviors in the medicinal leech. J Comp Neurol 402:168-180.

Rink TJ, Tsien RY, Warner AE (1980) Free calcium in Xenopus laevis embryos measured with ion selective microelectrodes. Nature 283:658-660.

Roerig B, Feller MB (2000) Neurotransmitters and gap junctions in developing neural circuits. Brain Res Rev 32:86-114.

Ruthazer ES, Akerman CJ, Cline HT (2003) Control of axon branch dynamics by correlated activity in vivo. Science 301:66-70.

Sawada M, Wilkinson JM, McAdoo DJ, Coggeshall RE (1976) The identification of 2 inhibitory cells in each segmental ganglion of the leech and studies on the ionic mechanism of the inhibitory junctional potentials produced by these cells. J Neurobiol 7:435-445.

Shepherd D, Murphey RK (1986) Competition regulates the efficacy of an identified synapse in crickets. J Neurosci 6:3152-3160.

Sillar KT, Simmers AJ (1994) Electrical coupling and intrinsic neuronal oscillations in rana temporaria spinal cord. Eur J Morphol 32:293-298.

Stuart AE (1970) Physiological and morphological properties of motoneurones in the central nervous system of the leech. J Physiol (Lond) 209:627-646.

Szabo TM, Faber DS, Zoran MJ (2004) Transient electrical coupling delays the onset of chemical neurotransmission at developing synapses. J Neurosci 24:112-120.

Taylor AL, Cottrell GW, Kleinfeld D, Kristan Jr WB (2003) Imaging reveals synaptic targets of a swim-terminating neuron in the leech CNS. J Neurosci 23:11402-11410.

Tresch MC, Kiehn O (2000) Motor coordination without action potentials in the mammalian spinal cord. Nat Neurosci 3:593-599.

Tresch MC, Kiehn O (2002) Synchronization of motor neurons during locomotion in the neonatal rat: predictors and mechanisms. J Neurosci 22:9997-10008.

Walton KD, Navarrete R (1991) Postnatal changes in motoneuron electrotonic coupling studied in the in vitro rat lumbar spinal cord. J Physiol (Lond) 433:283-306.

Ye M, Haralick RM (2000) Image flow estimation using facet model and covariance propagation. In: Vision interface: real world applications of computer vision, Vol 35 (Cheriet M, Yang YH, eds), pp 209-241. Hackensack, NJ: World Scientific.

Zoccolan D, Torre V (2002) Using optical flow to characterize sensorymotor interactions in a segment of the medicinal leech. J Neurosci 22: 2283-2298. 